\title{
The Recovery Agenda: The Shared Role of Peers and Professionals
}

\author{
William L. White, MA, ${ }^{1}$ \\ Arthur C. Evans, Jr., $\mathrm{PhD}^{2}$
}

\begin{abstract}
The alcohol and other drug (AOD) problems arena is extending its organizing center from knowledge drawn from the study of addiction-related pathologies and clinical and social interventions to knowledge drawn from the lived experience of long-term addiction recovery. A distinctive element within this shift is the increased use of non-clinical, peer recovery support services as an adjunct or alternative to specialized, professionally directed addiction treatment. This paper reviews the context of this shift, notes the evolution from competing to integrated service models, and outlines a decade of experience integrating peer recovery support services within the City of Philadelphia's behavioral healthcare system. Integrated models of peer-professional addiction recovery support have the potential of capitalizing on the respective strengths of clinical and environmental interventions into severe AOD problems.
\end{abstract}

Key Words: Addiction, recovery management, recovery-oriented systems of care, recovery support services, recovery community institutions

Recommended Citation: White WL, Evans AC. The recovery agenda: the shared role of peers and professionals. Public Health Reviews. 2014;35: epub ahead of print.

\section{INTRODUCTION}

Recovery is emerging as a new organizing construct within the alcohol and other drug (AOD) problems arena in the United States ${ }^{1,2}$ and the United Kingdom. ${ }^{3,4}$ There is growing consensus that policies based on research of drug-related pathologies and evaluative studies of clinical and social interventions can be substantially enriched by drawing lessons from the lived

\footnotetext{
${ }^{1}$ Chestnut Health Systems, Punta Gorda, FL, USA.

${ }^{2}$ Philadelphia Department of Behavioral Health and Developmental disAbility Services, Philadelphia, PA, USA.
}

Corresponding Author Contact Information: William White at bwhite@ chestnut.org; 3329 Sunset Key Circle, \# 203, Punta Gorda, FL 33955, USA. 
solutions to these problems at personal, family, neighborhood, and community levels. Reflecting a parallel shift from pathogenic (disease) to salugenic (wellness) approaches in medicine, psychology, and criminology, 5,6 the emergence of a recovery paradigm is also being influenced by the growth and diversification of recovery mutual aid societies and new recovery support institutions (e.g., recovery community centers, recovery homes, recovery schools, recovery industries, recovery ministries), the emergence of an ecumenical culture of recovery embracing secular, spiritual, and religious pathways of recovery, and the political mobilization of individuals and families in recovery via US- and UK-based recovery advocacy movements. ${ }^{7-9}$ Also influential have been the reconceptualization of severe AOD problems as chronic disorder ${ }^{10,11}$ and accompanying calls to shift acute and palliative care models of addiction treatment to models of assertive and sustained recovery management (RM) nested within larger recovery-oriented systems of care (ROSC). ${ }^{12,13}$ The quickening pace of service integration initiatives under the influence of unfolding healthcare reforms and suggestions that recovery might serve as a conceptual bridge for integrating mental health, addiction treatment, and primary healthcare have also added impetus toward a recovery policy agenda. ${ }^{14-16}$

The increasing prominence of recovery as an organizing construct is reflected in national AOD policy and funding initiatives, ${ }^{17}$ multi-national efforts to define recovery, ${ }^{18-20}$ state and local RM and ROSC experiments, ${ }^{21}$ and increasing calls for a recovery research agenda that can guide the transformation of traditional models of intervention toward the RM and ROSC visions. ${ }^{22}$ This emerging recovery agenda is being further influenced by advocacy to integrate person-centered clinical models of intervention with population-centered public health models of intervention across the dichotomized worlds of abstinence and harm reduction. ${ }^{23-25} \mathrm{RM}$ and ROSC implementation has progressed for nearly a decade in selected states and cities, but remains in most areas a nascent movement whose promises have yet to be tested in diverse geographical, cultural and political contexts.

A central component of RM and ROSC initiatives has been the inclusion of non-clinical, most often peer-based, recovery support services across the stages of long-term recovery. ${ }^{26-28}$ The purpose of this paper is to review the emergence of peer recovery support services as a more visible component of community responses to AOD problems, describe how professional and peer services are being integrated within the City of Philadelphia's behavioral health systems transformation process, and highlight lessons learned from the Philadelphia experience. 


\section{DISCUSSION}

\section{Professional and Peer Models of Addiction Recovery Support}

Support for the resolution of AOD problems often involves the sequential mobilization of intrapersonal resources; family, extended family, and social network resources; generalist helping resources within the community; and for the most severe and complex AOD problems, specialized addiction recovery mutual aid and/or professional treatment resources. The relationship between recovery mutual aid and professional treatment organizations has vacillated between periods of tension, separation, competition, and conflict and periods of reconciliation, reciprocal linkage, and experiments in collaboration and integration. ${ }^{29}$ People recovering from substance use disorders (SUDs) played prominent roles in the rise of modern addiction treatment, once making up nearly 70 percent of the addiction treatment workforce. Such representation has since dropped to approximately 30 percent following more than four decades of professionalization within the addiction counseling and addiction medicine workforce. ${ }^{28}$

The current growth of recovery support services (RSS) and related programs (e.g., alumni services, volunteer programs) marks, in part, a visible return of people in recovery into the addiction treatment milieu. RSS represent a new category of specialized resources - not treatment and not purely mutual aid - that link and supplement traditional recovery mutual aid and addiction treatment. RSS have gained prominence in the US due to their promotion and funding through the Center for Substance Abuse Treatment's Recovery Community Services Program (since 1998) and Access to Recovery program (since 2004). ${ }^{30}$ Their growth also reflects the broader integration of people credentialed by experience into helping roles within multiple service sectors (e.g., public health, primary medicine, mental health, child welfare)..$^{31-33}$

The term recovery support services sometimes refers to the whole spectrum of services that can support addiction recovery, but is most often applied to nonclinical services-supports beyond the arenas of clinical assessment, diagnosis, and treatment. ${ }^{26,34}$ The term peer recovery support services (PRSS) explicitly refers to RSS provided by persons in personal/ family recovery and who may possess additional qualities (e.g., age, gender, ethnicity, sexual orientation, combat experience, past incarceration) that enhance the process of mutual identification with the recovery support relationship. Recovery coaching, presently delivered in both peer and professional formats in the US and the UK, is a subset of RSS that are focused on mentoring individuals into the recovery process and guiding the development of a recovery-based lifestyle in the community. ${ }^{28}$ 
PRSS flow from theoretical and conceptual underpinnings (e.g., experiential knowledge, wounded healer, helper principle) that are distinct from clinical models of addiction treatment. ${ }^{35-37}$ PRSS are delivered across the stages of pre-recovery engagement, recovery initiation and stabilization, recovery maintenance, and enhanced quality of personal/family life in long-term recovery (including efforts to break intergenerational cycles of problem transmission) - a far greater span of involvement than that seen within acute care models of addiction treatment. ${ }^{28}$

Where the primary unit of service within traditional addiction treatment is the individual, RSS encompasses the individual, family, social network, and community. The scope of RSS for individuals and families can include a very broad range of activities: recovery-focused outreach; assertive linkage to addiction treatment, recovery mutual aid, and other recovery community institutions; linkage to recovery-conducive housing, healthcare, child care, transportation, legal advocacy, education, employment, leisure activities; recovery mentoring/coaching (including stage-appropriate recovery education); recovery support group facilitation; post-treatment recovery checkups; and when needed, early re-intervention. Community level interventions include recovery resource mapping, recovery community mobilization and resource development, enhancement of recovery orientation within community service systems, and recovery-focused community education and advocacy. ${ }^{34,38}$

RSS are delivered within both paid and volunteer service models and are being delivered in a wide variety of organizational environments (including addiction treatment programs, recovery community organizations, faithbased organizations, managed behavioral healthcare organizations, allied health and human service organizations, and criminal justice organizations) ${ }^{39}$ RSS are also being delivered in a wide variety of geographical and cultural contexts $^{40-43}$ and in both face-to-face and technology-based mediums (e.g., RSS delivered via internet and smart phones) ${ }^{44}$ In terms of the degree of integration of RSS with addiction treatment, RSS may serve as an adjunct or alternative to addiction treatment and other community recovery support systems. RSS are being provided in the context of addiction treatment in sequential models (professional care followed by RSS), parallel models (professional care and RSS provided simultaneously by the same or different organizations), and integrated models (treatment services and RSS provided by the same organization or highly coordinated multiagency teams).

Early research reviews on $\operatorname{RSS}^{27,28}$ acknowledge the paucity of research on the effects of RSS but note promising early outcomes related to the role of recovery residences and school-based RSS in elevating recovery and related outcomes. ${ }^{45,46}$ These reviews and related studies also note the value 
of particular RSS components such as use of peers in recovery to promote a peer help culture within addiction treatment units, recovery coaching, outreach and engagement, assertive linkage to recovery mutual aid and post-treatment recovery checkups, ${ }^{33,47,48}$ as well as the value and cost effectiveness of recovery coaching within particular service contexts. ${ }^{33,49}$ Formal evaluations of federal- and state-funded RSS also appear promising, ${ }^{50}$ with the State of Connecticut reporting dramatic reductions in costs by using RSS as an alternative to recycling individuals with high problem severity and low recovery capital through expensive episodes of acute detoxification and brief stabilization without measurable long-term recovery outcomes. The addition of RSS allowed the State of Connecticut to break this pattern, enhance recovery outcomes, and free up financial and human resources to serve a larger number of individuals and families. ${ }^{21}$

\section{The Philadelphia Experience with Professional/Peer RSS Integration}

RSS in Philadelphia are rooted in a recovery-focused behavioral health systems transformation process that began in 2005. ${ }^{51}$ Philadelphia has been involved in pioneering work in the treatment of addiction for more than a century. ${ }^{29,52}$ In 2005, new behavioral health leadership challenged local stakeholders to draw upon that deep tradition to forge a more recoveryfocused, person-centered system of care that could serve as a model for the country. The impetus for change was a series of town meetings and focus groups on the state of behavioral healthcare. These meetings generated consensus that acute and palliative care approaches to addiction treatment needed to be refocused toward sustained recovery management and expansion of the City's indigenous recovery support resources. This became a shared vision for both addiction treatment and mental health services, and PRSS became a central element within the transformation strategy led by the Department of Behavioral Health and Intellectual disAbility Services (DBHIDS). The transformation process challenged existing status hierarchies, expanded and reallocated financial resources, significantly broadened the spectrum of provider organizations and helper roles and required substantial and sustained education, training and technical assistance that continue to the present. The challenges of transformation and the change facilitation strategies employed have been detailed in an earlier report. ${ }^{51}$

DBHIDS created an infrastructure through which PRSS could be designed, implemented, and evaluated. That infrastructure included creation of a DBHIDS Recovery Advisory Council, increased recovery representation within DBHIDS staff and professional policy committees, and the design and delivery of a Recovery Foundations Training for multiple stakeholders 
within Philadelphia's behavioral healthcare system (83 workshops, 2,490 participants). A DBHIDS transformation blueprint and Office of Addiction Services (OAS) strategic plan, developed with intense and sustained involvement of numerous community stakeholders, led to a sustained partnership between DBHIDS and the Pennsylvania Recovery Organization - Achieving Community Together (PRO-ACT) — the leading local recovery advocacy organization, the opening of Philadelphia's first recovery community center, and the subsequent expansion of PRSS. To date (20082013), more than 5,400 individuals have been provided PRSS through the recovery community center. Evaluations of the effects of the more than 39,000 hours of PRSS delivered through the recovery community center reveal enhanced abstinence outcomes as well as increases in employment, educational involvement income, housing stability and parental custody of children. In addition to providing system-wide and program-level guidance on service design, peers have been used to conduct street outreach, provide support during treatment, solicit feedback from clients following treatment on the relative quality of their treatment experience, and provide sustained post-treatment monitoring and support.

PRSS were nested within larger efforts to culturally mobilize diverse communities of recovery for service and advocacy. Such efforts were guided by imbedding recovery prevalence questions within existing community health surveys and mapping recovery support resources by city census tracts. Mobilization activities between 2005 and 2013 included hosting national speakers to address local communities of recovery about recovery advocacy and peer recovery support initiatives, a 12-week peer leadership academy (59 graduates in 2011-2012), 34 Certified Peer Specialist (peers in paid service roles) trainings (611 trainees), 92 recovery storytelling workshops (1,532 trainees), 31 peer group facilitator trainings (519 trainees), and 285 Taking Recovery to the Streets presentations to individuals in treatment provided by 59 trained peers. Through support of a federal Access to Recovery grant, DBHIDS expanded recovery support services through a network of 52 community and faith-based service providers with service coordination provided by 22 Peer Recovery Specialists. More than 8,500 individuals were served through this initiative between 2011 and 2013. Since 2005 DBHIDS has also sponsored two major professional/peer recovery conferences, co-sponsored Amends in Action events (involvement of people in recovery in community service projects), and co-hosted an annual public recovery celebration event (with more than 18,000 participants in 2012). Other efforts to increase the visibility of recovery in Philadelphia included support for recovery-focused sports, music, radio, television, and journalism activities, and creating recovery murals as part of Philadelphia's Mural Arts Program. 
DBHIDS also took strategic steps to increase the recovery orientation of its treatment providers. These steps included encouraging treatment programs to develop consumer councils, developing and disseminating recoveryfocused practice guidelines, providing enhanced service reimbursement rates for achieving recovery-focused service benchmarks, and creating multiple ongoing opportunities for communication and collaboration between leaders of recovery community organizations, addiction treatment programs, and other indigenous community service programs (e.g., faith-based recovery ministries).

\section{NET: A Peer-Professional Integration Case Study}

The NorthEast Treatment Centers' (NET) development of PRSS illustrates how the transformation process influenced the culture of Philadelphia's network of addiction treatment providers. Founded in 1970, NET is a nonprofit organization offering addiction, mental health, foster care, and other social services to adults, adolescents, children, and their families in Southeastern Pennsylvania and the state of Delaware. With an annual operating budget of approximately USD \$59 million, Net's staff of more than 900 employees serves more than 9,000 individuals and their families each year. NET's addiction-related service menu includes intensive outpatient services $(3,800+$ per year), residential services $(250+$ per year), medically monitored inpatient detoxification $(2,100+$ per year), and medication-assisted treatment (500+ per year).

In response to the larger recovery-focused systems transformation process sparked by the Philadelphia DBHIDS, NET embarked on its own internal systems transformation process in December of 2005. NET's transformation process included four key steps: 1) involving all NET stakeholders in discussions about the shift toward an RM and ROSC service philosophy, 2) using training and clinical supervision to help staff and peer specialists redefine roles and service relationships, 3) merging fragmented and separate program services into an integrated continuum of care, and 4) creating a Consumer Council within each of four service delivery sites and a NET advisory board (with representation from each Consumer Council) to drive NET's organizational transformation process and the integration of PRSS. ${ }^{53}$ The Consumer Councils meet weekly, host a monthly recovery recognition day and annual recovery celebration and awards dinner, and publish newsletters (e.g., the bi-monthly NET-Steps) that are disseminated to all NET consumers and staff. The Councils also oversee the NET volunteer program, conduct client community integration workshops, oversee the consumer speaker's bureau, co-staff four recovery community 
centers (consumer-operated drop-in centers that provide pre-treatment, in-treatment and post-treatment social support, emergency housing, treatment referral, medical referral, free clothing, job coaching, life skills training, and a computer lab), and conduct their own fundraising activities.

Philadelphia's recovery-focused transformation process provided an opportunity for NET to significantly increase its peer recovery specialist positions. Currently, 21 peer specialists (19 full time equivalents) are employed at NET - funded out of increased revenues NET receives from the enhanced retention and service participation rates that were a direct result of PRSS.${ }^{54}$ NET's paid PRSS positions are supplemented by a cadre of peer volunteers called peer mentors drawn from current and former NET service consumers.

Peer specialists and volunteer peer mentors collectively perform multiple functions at NET. They conduct street outreach and link people in need to treatment and recovery mutual aid resources, greet and orient all new NET clients and their families, offer daily encouragement for participation in treatment and recovery support activities, mediate conflict between staff and clients, and make face-to-face or telephonic contact with any client who misses a service appointment. They co-facilitate (with professional staff) recovery planning groups and facilitate recovery support groups.

\section{RECOMMENDATIONS}

Based on the Philadelphia experience integrating PRSS with professionaldirected addiction treatment, we would offer the following observations and recommendations to others embarking on similar efforts.

Potential for Successful Peer-Professional Integration. PRSS can be successfully integrated with professional models of care (e.g., blended and sequenced in ways that potentially magnify the catalytic potency of each). This can occur at all levels of behavioral healthcare systems, but such integration requires planning, education, and support for peers and professionals as well as education of the larger community about the role and value of PRSS.

Models of Implementation. PRSS are being added to behavioral health service systems in three sometimes sequential patterns: as an encapsulated appendage/adjunct to professional services, as part of the transformation of a particular system component, or integrated throughout a systems-wide transformation process ${ }^{51}$ It is our experience that the third of these choices is the far superior option. The failure to provide such integration can inadvertently lead to competition, conflict, and disaffection among both peers and professionals. 
Structure for Integrating PRSS. The design and delivery of PRSS are marked by different theoretical foundations, service elements, service delivery environments, and more nuanced ethical guidelines and cultural etiquette governing service relationships than those that have traditionally guided professionally directed addiction treatment. ${ }^{28}$ As a result, PRSS require different approaches to recruitment, selection, orientation/training, and supervision than those used for professional staff. Care must be taken in the integration process not to de-professionalize clinical services or professionalize peer support relationships. To effectively guide ROSC transformation and PRSS integration, we recommend use of a task force or advisory group that contains peer, professional, and broader community representation as well as connection to expertise within other communities that have successfully implemented PRSS.

Authenticity of Recovery Representation. Service consumers and their families as well as representatives from local communities of recovery can and should play significant roles in decision-making at all levels of the behavioral healthcare system, including the design, delivery, and evaluation of day-to-day recovery support activities. When involving people in recovery within ROSC and PRSS planning processes and recruiting PRSS specialists in staff or volunteer roles, we recommend cultivating to the extent possible a diversity of recovery representation across pathways of recovery, family as well as personal recovery representation, people in different stages of recovery, and representation across the boundaries of age, gender, and culture. It is also important to assure authenticity of recovery voices, e.g., avoiding potential problems of double agentry by selecting individuals to represent the recovery experience who do not have other personal, professional, or institutional conflicts of interest.

Balance of PRSS Functions. PRSS involve three linked functions: 1) supporting personal and family health across the stages of long-term recovery, 2) expanding community recovery capital by helping create the physical, psychological, and social space in the community within which recovery can flourish, and 3) advocating local, state, and national policies that promote health and full community reintegration of individuals and families in recovery. Achieving a balance of focus across these three arenas of action moves PRSS beyond traditional clinical models of intervention toward ecological models of community development and cultural renewalwhat the authors have depicted as community recovery. ${ }^{55}$

PRSS and Stages of Recovery. Where the majority of traditional addiction treatment resources are allocated to recovery initiation and stabilization, PRSS have the potential of shortening addiction careers through prerecovery identification, engagement, and recovery priming. PRSS also have 
the potential for lowering treatment dropout rates and post-treatment SUD recurrence through the provision of in-treatment recovery support and posttreatment support of the transitions from recovery initiation to recovery maintenance and to enhanced quality of personal and family life in longterm recovery. The latter include family-, parental- and child-focused supports aimed at breaking intergenerational cycles of problem transmission. It is at this level that PRSS find common ground and partnership with community-level primary prevention and early intervention programs.

Critical Need for PRSS Research. The rapid implementation of PRSS in the US and UK is far ahead of the research on PRSS. Many critical questions related to PRSS remain unanswered or only partially answered, including most importantly: 1) Do PRSS enhance personal long-term recovery outcomes? 2) What are the effects of family-focused PRSS on the family and family subsystems? 3) Do recovery outcomes differ by the characteristics of who is providing the RSS (e.g., recovery versus non-recovery status) or by the organizational setting through which PRSS are delivered, e.g., treatment organization, recovery community organization, managed behavioral healthcare organization, faith-based organization? 4) What "active ingredients" of PRSS exert the greatest influence on recovery outcomes and system cost outcomes? 5) Are there particularly potent combinations and sequences of professional treatment and PRSS as measured by longterm recovery outcomes? 6) Do such effective service combinations and sequences differ across particular populations, including those who have a history of consuming inordinate proportions of system resources without measureable recovery outcomes? 7) Can PRSS saturation alter problem prevalence and recovery prevalence at a community level?

PRSS Financing. Peer recovery support services are being financed through a variety of mechanisms, including federal and state grants, public and private fee-for-service contracts, and indirect financing through increased income from improved rates of recruitment, engagement, and retention (at a program level) and reduced rates of recidivism in acute levels of care (at a systems or payor level). We recommend that PRSS delivery sites cultivate a balanced payor mix and a safety net of peer volunteers to assure sustainability of recovery support.

Preventing Potential Iatrogenic Effects of PRSS. Given the long record of harm in the name of help within the history of clinical and social interventions into AOD problems, ${ }^{56,57}$ it is incumbent on behavioral health leaders to ask whether any inadvertent injuries could flow from the implementation of PRSS. In our experience with PRSS over the past decade in Philadelphia, such injuries could most likely occur in three areas. The first is potential exploitation and harm to peers related to excessive work demands, inadequate 
compensation, and inadequate support to assure physical safety and ameliorate work-related emotional distress. The second risk involves harm to clients that could come from poor boundary management within the peer service relationship (e.g., emotional, sexual, financial exploitation). The third risk is a more subtle and prolonged harm that could come to the community by potential erosion of the volunteer service ethic within communities of recovery that could result from the proliferation of paid recovery support roles. We have tried to address the former through development of ethical guidelines and processes of complaint and redress related to PRSS $^{58}$ and to prevent the latter by emphasizing that PRSS must supplement and not replace natural recovery supports that currently exist within the community.

\section{SUMMARY}

With appropriately timed professional and peer support, people addicted to alcohol and other drugs can initiate and maintain recovery under the most extreme and adverse conditions and, through that recovery process, also help bring healing and health to their families and neighborhoods. RSS, and more specifically PRSS, are emerging as a key strategy in transforming acute care and palliative care models of addiction treatment into models of assertive recovery management that enhance the scope and quality of recovery support while building the community recovery capital that can support long-term recovery from addiction and related problems. PRSS provide a window of opportunity to integrate clinical and public health models of AOD problem resolution, but considerable care must be taken in implementing such models and evaluating such new service mechanisms to assure optimal outcomes and prevent any unforeseen consequences of this shift in service delivery design.

\section{Acronyms List:}

$\mathrm{AOD}=$ alcohol and other drug

DBHIDS $=$ Department of Behavioral Health and Intellectual disAbility Services

NET $=$ NorthEast Treatment Centers

PRSS $=$ peer recovery support services

$\mathrm{RM}=$ recovery management

ROSC $=$ recovery-oriented systems of care

RSS $=$ recovery support services

SUD $=$ substance use disorder

Acknowledgements: Development of this paper was supported by the Philadelphia Department of Behavioral Health and Developmental disAbility Services. Brooke Feldman assisted in collection of data on DBHIDS peer recovery support activities.

Conflicts of Interest: None declared. 


\section{REFERENCES}

1. Clark HW. Recovery as an organizing concept. In: White, WL. Perspectives on Systems Transformation: How Visionary Leaders are Shifting Addiction Treatment toward a Recovery-Oriented System of Care. Chicago (IL): Great Lakes Addiction Technology Transfer Center; 2007. p.7-21.

2. White WL. Recovery: old wine, flavor of the month or new organizing paradigm? Subst Use Misuse. 2008;43:1987-2000.

3. Berridge V. The rise, fall, and revival of recovery in drug policy. Lancet. 2012; 379:22-3.

4. Wardle I. Five years of recovery: December 2005 to December 2010 - from challenge to orthodoxy. Drugs: Education, Prevention Policy. 2012;19:294-8.

5. Krentzman AR. Review of the application of positive psychology to substance use, addiction, and recovery research. Psychol Addict Behav. 2013;27:151-65.

6. Ronel N, Elisha E. A different perspective: introducing positive criminology. Int J Offender Ther Comp Criminol. 2011;56:305-25.

7. Humphreys K. Circles of Recovery: Self-Help Organizations for Addictions. Cambridge: Cambridge University Press; 2004.

8. White WL. The new recovery advocacy movement in America. Addiction. 2007;102:696-703.

9. White WL, Kelly JF, Roth JD. New addiction recovery support institutions: mobilizing support beyond professional addiction treatment and recovery mutual aid. J Groups Addict Recover. 2012;7:297-313.

10. Dennis ML, Scott CK. Managing addiction as a chronic condition. Addict Sci Clin Pract. 2007;4:45-55.

11. McLellan AT, Lewis DC, O'Brien CP, Kleber HD. Drug dependence, a chronic medical illness: implications for treatment, insurance, and outcomes evaluation. JAMA. 2000;284:1689-95.

12. Kelly JF, White WL. Addiction Recovery Management: Theory, Science and Practice. New York (NY): Springer Science; 2011.

13. White WL. Recovery management and recovery-oriented systems of care: scientific rationale and promising practices. Pittsburgh (PA): Northeast Addiction Technology Transfer Center, Great Lakes Addiction Technology Transfer Center, Philadelphia Department of Behavioral Health \& Mental Retardation Services; 2008.

14. Buck JA. The looming expansion and transformation of public substance abuse treatment under the Affordable Care Act. Health Aff. 2011;30:1402-10.

15. Davidson L, White W. The concept of recovery as an organizing principle for integrating mental health and addiction services. J Behav Health Serv Res. 2007;34:109-20.

16. Gagne CA, White W, Anthony WA. Recovery: a common vision for the fields of mental health and addictions. Psychiatr Rehabil J. 2007;32:32-7.

17. Center for Substance Abuse Treatment (CSAT). National Summit on Recovery: conference report. DHHS Publication No. (SMA) 07-4276. Rockville (MD): Substance Abuse and Mental Health Services Administration; 2007. 
18. Betty Ford Institute Consensus Panel. What is recovery? A working definition from the Betty Ford Institute. J Subst Abuse Treat. 2007;33:221-8.

19. Scottish Government. The road to recovery: a new approach to tackling Scotland's drug problem. Edinburgh: Scottish Government; 2008.

20. United Kingdom Drug Policy Commission. A consensus definition of recovery. 2008. Available from URL: http://www.ukdpc.org.uk/resources/A\%20Vision \%20of\%20Recovery.pdf (Accessed 24 June 2010).

21. Kirk T. Connecticut's journey to a statewide recovery-oriented health-care system: Strategies, successes and challenges. In: Kelly JF, White WL, (editors). Addiction Recovery Management: Theory, Science and Practice. New York (NY): Springer Science; 2011. p.209-34.

22. Laudet A, Flaherty M, Langer D. Building the science of recovery. Pittsburgh (PA): Institute for Research, Education and Training and Northeast Addiction Technology Transfer Center; 2009.

23. Hunt N. Recovery and harm reduction: time for a shared, development-oriented, programmatic approach? In: Pates R, Riley D, (editors). Harm Reduction in Substance Use and High-Risk Behaviors. Chichester: Wiley-Blackwell; 2012.

24. Kellogg SH. On "gradualism" and the building of the harm reductionabstinence continuum. J Subst Abuse Treat. 2003;25:241-7.

25. White WL, Mojer-Torres L. Recovery-oriented methadone maintenance. Chicago (IL): Great Lakes Addiction Technology Transfer Center, Philadelphia Department of Behavioral Health and Mental Retardation Services and Northeast Addiction Technology Transfer Center; 2010.

26. Kaplan L. The role of recovery support services in recovery-oriented systems of care. DHHS Publication No. (SMA) 08-4315. Rockville (MD): Center for Substance Abuse Services, Substance Abuse and Mental Health Services Administration; 2008.

27. Laudet AB, Humphreys K. Promoting recovery in an evolving policy context: what do we know and what do we need to know about recovery support services? J Subst Abuse Treat. 2013;45:126-33.

28. White WL. Peer-based addiction recovery support: history, theory, practice, and scientific evaluation. Chicago (IL): Great Lakes Addiction Technology Transfer Center and Philadelphia Department of Behavioral Health and Intellectual disAbility Services; 2009.

29. White WL. Slaying the dragon: the history of addiction treatment and recovery in America. Bloomington (IL): Chestnut Health Systems, 1998.

30. Kaplan L, Nugent C, Baker M, Clark HW, Veysey BM. Introduction: the Recovery Community Services Program. Alcohol Treat Q. 2010;28:244-55.

31. Brownstein JN, Bone LR, Dennison C, Hill MN, Kim M, Levine DM. Community health workers as interventionists in the prevention and control of heart disease and stroke. Am J Prev Med. 2005;29:128-33.

32. Mowbray CT, Moxley DP, Jasper CA, Howell LL. Consumers as Providers in Psychiatric Rehabilitation. Columbia (MD): International Association of Psychosocial Rehabilitation Services (IAPSRS); 1997. 
33. Ryan J, Choi S, Hong JS, Hernandez P, Larrison CR. Recovery coaches and substance exposed births: an experiment in child welfare. Child Abuse Negl. 2008;32:1072-9.

34. Center for Substance Abuse Treatment. What are peer recovery support services? HHS Publication No. (SMA) 09-4454. Rockville (MD): Substance Abuse and Mental Health Services Administration, U.S. Department of Health and Human Services; 2009.

35. Borkman T. Experiential knowledge: a new concept for the analysis of self-help groups. Soc Serv Rev. 1976;50:445-56.

36. Jackson SW. The wounded healer. Bull Hist Med. 2001;75:1-36.

37. Riessman F. The "helper" therapy principle. Soc Work. 1965;April:27-32.

38. Valentine P. Peer-based recovery support services within a recovery community organization: the CCAR experience. In: Kelly JF, White WL, (editors). Addiction Recovery Management: Theory, Science and Practice. New York (NY): Springer Science; 2011. p.259-80.

39. White W, Humphreys K, Bourgeois M, Chiapella P, Evans A, et al. The status and future of addiction recovery support services in the United States: report of the BFI/UCLA Consensus Conference on Recovery Support Services; 2013.

40. Achara-Abrahams I, Evans AC, Ortiz J, Lopez Villegaz D, O’Dell J, et al. Recovery management and African Americans: a report from the field. Alcohol Treat Q. 2012;30:263-92.

41. Evans AC, Achara I, Lamb R, White W. Ethnic-specific support systems as a method for sustaining long-term addiction recovery. J Groups Addict Recover. 2012;7:171-88.

42. Moore $S$, Coyhis D. The multicultural wellbriety peer recovery support program: two decades of community-based experience. Alcohol Treat Q. 2010;28:27392 .

43. Veysey BN, Grasmere J, Andersen R. Supporting peer recovery in rural New England: the RECOVER Project, Franklin County, MA. Alcohol Treat Q. 2010;28:306-25.

44. Klein AA, Slaymaker VJ, Dugosh KL, McKay JR. Computerized continuing care support for alcohol and drug dependence: a preliminary analysis of usage and outcomes. J Subst Abuse Treat. 2012;37:435-42.

45. Cleveland HH, Harris KS, Baker AK, Herbert R, Dean LR. Characteristics of a collegiate recovery community: maintaining recovery in an abstinencehostile environment. J Subst Abuse Treat. 2007;33:13-23.

46. Jason LA, Mericle AA, Polcin DL, White WL, the National Association of Recovery Residences. A primer on recovery residences in the United States. Atlanta (GA): National Association of Recovery Residences; 2012.

47. Dennis ML, Scott CK. Four-year outcomes from the Early Re-Intervention (ERI) experiment using Recovery Management Checkups (RMCs). Drug Alcohol Depend. 2012;121:10-17.

48. Galanter M, Castaneda R, Salamon I. Institutional self-help therapy for alcoholism: clinical outcome. Alcohol Clin Exp Res. 1987;11:424-9 
49. Humphreys K, Moos R. Can encouraging substance abuse patients to participate in self-help groups reduce demand for health care? A quasi-experimental study. Alcohol Clin Exp Res. 2001;25:711-16.

50. Krupski A, Campbell K, Joesch JM, Lucenko BA, Roy-Byrne P. Impact of access to recovery services on alcohol/drug treatment outcomes. J Subst Abuse Treat. 2009;37:435-42.

51. Achara-Abrahams I, Evans AC, King JK. Recovery-focused behavioral health systems transformation: a framework for change and lessons learned from Philadelphia. In: Kelly JF, White WL, (editors). Addiction Recovery Management: Theory, Science and Practice. New York (NY): Springer Science; 2011. p.187-208.

52. Leonard EC. The treatment of Philadelphia inebriates. Am J Addict. 1997;6:1-10.

53. The Net Consumer Council, Evans AC, Lamb RC, Mendelovich S, Schultz CJ, White WL. The role of clients in a recovery-oriented system of addiction treatment: the birth and evolution of the NET Consumer Council. Philadelphia (PA): Philadelphia Department of Behavioral Health and Mental Retardation Services; 2007.

54. McSherry T. The recovery-focused transformation of addiction treatment: an interview with Terence McSherry. 2012. Available from URL: http://www. williamwhitepapers.com/pr/2012\%20Terence\%20McSherry\%20Interview.pdf (Accessed 9 July 2013).

55. Evans AC, Lamb R, White WL. The community as patient: recovery-focused community mobilization in Philadelphia, 2005-2012. Alcohol Treat Q. in press.

56. Ilgen MA, Moos R. Deterioration following alcohol use disorder treatment in Project MATCH. J Stud Alcohol. 2005;66:517-25.

57. White WL, Kleber HD. Preventing harm in the name of help: a guide for addiction professionals. Counselor. 2008;9:10-17.

58. White W, the PRO-ACT Ethics Workgroup, with legal discussion by Popovits R. \& Donohue B. Ethical guidelines for the delivery of peer-based recovery support services. Philadelphia: Philadelphia Department of Behavioral Health and Mental Retardation Services; 2007. 